\title{
Continuous ESP Block Technique for an Open Thoracotomy in Neurofibromatosis Patient
}

\author{
Matheus de Castro Abi-Ramia Chimelli, Amanda Caroline Figueiredo Gomes Andrade, \\ Marcelo Grisólia Gonçalo, Clarissa Mayara de Sales Guimarães, \\ Carlos Frederico Panisset Lanhas La Cava, Carlos Darcy Alves Bersot
}

Department of Anesthesiology, Hospital Federal da Lagoa, Rio de Janeiro, Brazil

Email address:

matheuscarchimelli@gmail.com (M. de C. A. Chimelli)

\section{To cite this article:}

Matheus de Castro Abi-Ramia Chimelli, Amanda Caroline Figueiredo Gomes Andrade, Marcelo Grisólia Gonçalo, Clarissa Mayara de Sales Guimarães, Carlos Frederico Panisset Lanhas La Cava, Carlos Darcy Alves Bersot. Continuous ESP Block Technique for an Open Thoracotomy in Neurofibromatosis Patient. Advances in Surgical Sciences. Vol. 7, No. 2, 2019, pp. 35-37. doi: 10.11648/j.ass.20190702.12

Received: November 9, 2019; Accepted: December 17, 2019; Published: December 30, 2019

\begin{abstract}
Neurofibromatosis remains a challenge for the anesthesiologist as it brings issues especially regarding safety of anesthetic management such as airway management and neuraxial anesthesia. We report a case of a 60 year-old woman with controlled clinical comorbidities and neurofibromatosis, admitted for an open thoracotomy for excision of a growing pulmonary lesion, highly suspicious for malignancy. She had cutaneous tumors covering the majority of her skin. No neuroimage was available to exclude neuraxial or intracranial tumors, ensuring the safety for neuraxial anesthesia, therefore a combined technique was proposed with general anesthesia and a continuous erector spinae plane (ESP) block for anesthesia and analgesia. Surgery occurred successfully and there was no need to supplement or alter the initially planned anesthetic technique. Patient referred pain score ranging from 0-1 in rest and motion postoperative and required no complementary opioid for pain control. Infusion in the ESP catheter was maintained for $48 \mathrm{~h}$, after which it was removed and patient discharged without further complications or pain.
\end{abstract}

Keywords: ESP Block Technique, Thoracotomy, Neurofibromatosis Patient, Anesthesia

\section{Introduction}

Neurofibromatosis is an hereditary disease transmitted in an autosomal dominant fashion. It has common characteristics such as the formation of tumors originated by ectodermal and mesodermal tissues, most commonly skin neurofibromas and café-au-lait macules. It is divided two types: type 1 (or von Recklinghausen) and type 2, type 1 being the most common type, with an estimated incidence of 1 in 2500-3300 with a penetration of $100 \%$, meaning that every patient affected by this disease will present a clinical manifestation throughout its life [1-3].

Neurofibromatosis affects every major anatomical system and brings for the anesthesiologist important issues related, most importantly, to potential airway difficulty, tumors affecting peripheral nerves, and neuraxial or intracranial tumors [3, 4]. In the following case we discuss a patient with neurofibromatosis type 1 admitted for an open thoracotomy surgery for pulmonary tumor resection and the option for an alternate analgesic technique.

\section{Case Presentation}

A 60 year-old woman with clinically controlled systemic diseases (hypertension, hypothyroidism and depression) and neurofibromatosis was admitted for a resection of a pulmonary tumor suspected malignant through an open thoracotomy. The tumor was spiculated and had grown in the last year measuring $2,3 \times 1,9 \mathrm{~cm}$ in the superior lobe of the right lung in a chest $\mathrm{CT}$ image.

Her body was covered with cutaneous neurofibromas (Figures 1 and 2) and although her neurological exam had no alterations, she did not have a neurologic radiologic exam to rule out intracranial or neuraxial tumors.

In the operating room she was monitored with ECG, pulse oximeter, non-invasive blood pressure in the left arm and a venous puncture in the left arm with a 22 gauge peripheral 
catheter. She was sedated with midazolam and fentanyl and a direct radial artery blood pressure in the right arm was acquired as well as a central venous catheter was punctured in the right internal jugular vein. She was then placed in left lateral decubitus and a catheter was placed in the erector spinae plane (ESP) at T5 level guided and confirmed with ultrasound imaging and ropivacaine $0,5 \% 20 \mathrm{ml}$ dose was injected.

Following preoxygenation with $100 \%$ oxygen general anesthesia was induced with propofol $120 \mathrm{mg}$, lidocaine $80 \mathrm{mg}$, rocuronium $40 \mathrm{mg}$ and fentanyl $200 \mathrm{mcg}$. A doublelumen endotracheal RobertShaw type tube, 35FR was successfully placed aiming one-lung ventilation during surgery. Anesthesia was maintained with sevoflurane 1,0$1,5 \%$, oxygen and a continuous infusion of ropivacaine $0,5 \%$ $10 \mathrm{ml} / \mathrm{h}$ in the catheter inserted at the ESP.

Surgery occurred successfully without complications in 4 hours. Patient was extubated in the operating room and related no pain or other complaints before being admitted in the ICU for immediate postoperative care. She remained for $24 \mathrm{~h}$ in the ICU and then remained in the ward for $48 \mathrm{~h}$ before being discharged from the hospital.

Infusion in the ESP catheter was maintained with the same anesthetic solution for $48 \mathrm{~h}$ postoperatively and then the catheter was removed without complications. Patient referred pain score ranging from $0-1$ in motion and in rest, needing no complementary opioid for analgesic pain control and was discharged without further complications.

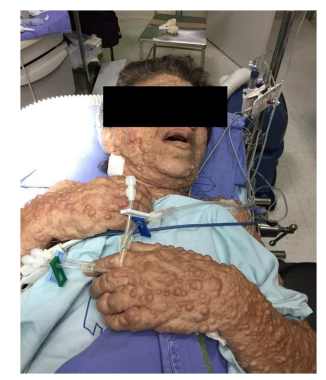

Figure 1. Patient with neurofibromas throughout her body.

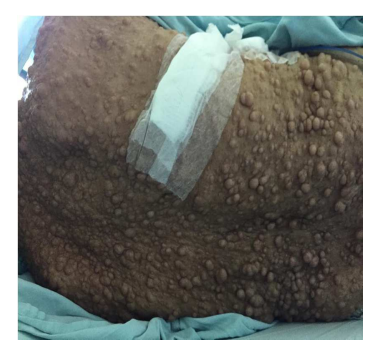

Figure 2. Patient with neurofibromas throughout her body.

\section{Discussion}

The erector spinae plane block was first described in 2016 in patients with chronic thoracic pain [5]. Through these years its use has been widespread and its efficacy and power as a substitute for neuraxial blocks is still in current discussion. However, Chin et al $[6,7]$ demonstrated promising results for abdominal surgery analgesia, reducing postoperative opioid use and its complications.

Neuraxial blocks (epidural or subdural) are still the standard analgesic blueprint especially to avoid opioid and its adverse effects and may also reduce morbidity after major abdominal and thoracic surgery [8]. Block et al [9] showed in a meta-analysis how superior local anesthetics in an epidural block are compared to parenteral opioids for postoperative analgesia in both abdominal and thoracic surgeries with almost no complications related.

Neurofibromatosis is a disease in which anesthesia and analgesia can be challenging. There is and increased incidence of epilepsy due to neurologic tumors [1,3] and increased risk of challenging airway management $[1,3,10]$, among other possible complications. Few studies regarding safety in neuraxial and peripheral blocks in these patients can be found, the majority of them are obstetric case reports [11, 12]. The risks of regional anesthesia in patients with neurofibromatosis rely basically on 2 major concerns: a theoretical spread of tumor cells and, most importantly, bleeding caused by manipulation of these tumors $[4,11,12]$. Therefore, those studies recommend a thorough neurological examination to discard possible previous alterations or intracranial and neuraxial tumors prior to regional anesthesia management $[4,11,12]$ and after the surgery also a thorough neurological examination to ensure that the patient has returned to his preexisting neurologic status [3] and the documentation of these findings.

The decision to avoid neuraxial anesthesia in our patient was guided by the absence of neuroimage ruling out tumors that could bring further complications to the patient. In that sense, a promising and safer choice of analgesia was offered, the ESP block, and in our case, successful pain management was achieved.

\section{Conclusion}

Neurofibromatosis is considered a rare condition, specially to the anesthesiologist routine practice. In that sense, there are few evidence regarding anesthetic management and its safety towards these patients. Patients undergoing major abdominal and thoracic surgery that are candidates to receive regional anesthesia should have a well-documented neurologic physical and radiologic examination allowing a safe choice of anesthetic and analgesic management for the patient, anesthesiologist and surgeon. ESP block still need to prove its role as an efficient primary choice of anesthetic and analgesic technique for the majority of population, and while it doesn't, it remains as an interesting alternative for patients with any type of relative or absolute contraindication for neuraxial anesthesia, as shown in the presented case.

\section{References}

[1] Hirsch NP, Murphy A, Radcliffe JJ. Neurofibromatosis: clinical presentations and anaesthetic implications. $\mathrm{Br} J$ Anaesth. 2001; 86: 555-564. 
[2] Crowe FW, Schull WJ, Neel JV. A Clinical, Pathological, and Genetic Study of Multiple Neurofibromatosis. Springfield, IL: Charles C. Thomas, 1956.

[3] Fox CJ, Tomaijian S, Kaye AJ, et al. Perioperative management of neurofibromatosis type 1. Ochsner J 2012; 12: $111-121$.

[4] McSwain JR, Doty JW, Wilson SW. Regional anesthesia in patients with pre-existing neurologic disease. Curr Opin Anesthesiol 2014; 27: 538-543.

[5] Forero M, Adhikary SD, Lopez H. The Erector Spinae Plane Block: A Novel Analgesic Technique in Thoracic Neuropathic Pain. Reg Anesth Pain Med 2016; 41: 621-627.

[6] Chin KJ, Adhikary S, Sarwani N, et al. The analgesic efficacy of pre-operative bilateral erector spinae plane (ESP) blocks in patients having ventral hernia repair. Anaesthesia 2017; 72: $1-8$.

[7] Chin KJ, Malhas L, Perlas A. The Erector Spinae Plane Block Provides Visceral Abdominal Analgesia in Bariatric Surgery. Reg Anesth Pain Med 2017; 42: 372-376.
[8] Freise H, Van Aken HK. Risks and Benefits of thoracic epidural anaesthesia. British Journal of Anaesthesia. 2011; 107 (6): 859-868

[9] Block BM, Liu SS, Rowlingson AJ, et al. Efficacy of Postoperative Epidural Analgesia: A Metanalysis. JAMA 2003; 290: 2455-2463.

[10] Williams UU, Zavala AM, Van Meter A, et al. Unanticipated Compression of the Trachea in a 5-month-old Undergoing an MRI for Evaluation of Neurofibromatosis. A\&A Case Reports 2017; 8: 1-3.

[11] Spiegel JE, Hapgood A, Hess PE. Epidural anesthesia in a parturient with neurofibromatosis type 2 undergoing cesarean section. International Journal of Obstetric Anesthesia. 2005; 14: 336-339.

[12] Esler MD, Durbridge J, Kirby S. Epidural haematoma after dural puncture in a parturient with neurofibromatosis. $\mathrm{Br} J$ Anaesth 2001; 87: 932-934. 